\title{
THE RANDOM WALK METHOD FOR INTERSECTING FAMILIES
}

\author{
NORIHIDE TOKUSHIGE
}

\begin{abstract}
Let $m(n, k, r, t)$ be the maximum size of $\mathscr{F} \subset\left(\begin{array}{c}{[n]} \\ k\end{array}\right)$ satisfying $\left|F_{1} \cap \cdots \cap F_{r}\right| \geq t$ for all $F_{1}, \ldots, F_{r} \in \mathscr{F}$. We report some known results about $m(n, k, r, t)$. The random walk method introduced by Frankl is a strong tool to investigate $m(n, k, r, t)$. Using a concrete example, we explain the method and how to use it.
\end{abstract}

\section{INTRODUCTION}

Let $n, k, r$ and $t$ be positive integers, and let $[n]=\{1,2, \ldots, n\}$. A family $\mathscr{G} \subset 2^{[n]}$ is called $r$-wise $t$-intersecting if $\left|G_{1} \cap \cdots \cap G_{r}\right| \geq t$ holds for all $G_{1}, \ldots, G_{r} \in \mathscr{G}$. Let us define a typical $r$-wise $t$-intersecting family $\mathscr{G}_{i}(n, r, t)$ and its $k$-uniform subfamily $\mathscr{F}_{i}(n, k, r, t)$, where $0 \leq i \leq\left\lfloor\frac{n-t}{r}\right\rfloor$, as follows:

$$
\begin{aligned}
\mathscr{G}_{i}(n, r, t) & =\{G \subset[n]:|G \cap[t+r i]| \geq t+(r-1) i\}, \\
\mathscr{F}_{i}(n, k, r, t) & =\mathscr{G}_{i}(n, r, t) \cap\left(\begin{array}{c}
n] \\
k
\end{array}\right) .
\end{aligned}
$$

Two families $\mathscr{G}, \mathscr{G}^{\prime} \subset 2^{[n]}$ are said to be isomorphic, and denoted by $\mathscr{G} \cong \mathscr{G}^{\prime}$, if there exists a vertex permutation $\tau$ on $[n]$ such that $\mathscr{G}^{\prime}=\{\{\tau(g): g \in G\}: G \in \mathscr{G}\}$.

Let $m(n, k, r, t)$ be the maximum size of $k$-uniform $r$-wise $t$-intersecting families on $n$ vertices. To determine $m(n, k, r, t)$ is one of the oldest problems in extremal set theory, which is still widely open. The case $r=2$ was observed by Erdős-Ko-Rado [6], Frankl [10], Wilson [30], and then $m(n, k, 2, t)=\max _{i}\left|\mathscr{F}_{i}(n, k, 2, t)\right|$ was finally proved by Ahlswede and Khachatrian [2]. Frankl [8] showed $m(n, k, r, 1)=\left|\mathscr{F}_{0}(n, k, r, 1)\right|$ if $(r-1) n \geq r k$. Partial results for the cases $r \geq 3$ and $t \geq 2$ are found in $[14,16,24$, 26, 27, 23, 29]. All known results suggest

$$
m(n, k, r, t)=\max _{i}\left|\mathscr{F}_{i}(n, k, r, t)\right| .
$$

Now we introduce the $p$-weight version of the Erdős-Ko-Rado theorem. Throughout this paper, $p$ and $q=1-p$ denote positive real numbers. For $X \subset[n]$ and a family $\mathscr{G} \subset 2^{X}$ we define the $p$-weight of $\mathscr{G}$, denoted by $w_{p}(\mathscr{G}: X)$, as follows:

$$
w_{p}(\mathscr{G}: X)=\sum_{G \in \mathscr{G}} p^{|G|} q^{|X|-|G|}=\sum_{i=0}^{|X|}\left|\mathscr{G} \cap\left(\begin{array}{c}
X \\
i
\end{array}\right)\right| p^{i} q^{|X|-i} .
$$

We simply write $w_{p}(\mathscr{G})$ for the case $X=[n]$, for example, we have $w_{p}\left(\mathscr{G}_{0}(n, r, t)\right)=p^{t}$.

Date: December 26, 2009, 09:38pm.

2000 Mathematics Subject Classification. Primary: 05D05 Secondary: 05C65.

Key words and phrases. Erdős-Ko-Rado Theorem; intersecting family; $p$-weight.

The author was supported by MEXT Grant-in-Aid for Scientific Research (B) 16340027. 
Let $w(n, p, r, t)$ be the maximum $p$-weight of $r$-wise $t$-intersecting families on $n$ vertices. It might be natural to expect

$$
w(n, p, r, t)=\max _{i} w_{p}\left(\mathscr{G}_{i}(n, r, t)\right) .
$$

Ahlswede and Khachatrian proved that this is true for $r=2$ in [3] (cf. [5, 7, 23]). This includes the Katona theorem [19] about $w(n, 1 / 2,2, t)$. It is shown in [15] that

$$
w(n, p, r, 1)=p \text { for } p \leq(r-1) / r .
$$

To state some more related results let us define some collections of families as follows.

$$
\begin{aligned}
\mathbf{G}(n, r, t) & =\left\{\mathscr{G} \subset 2^{[n]}: \mathscr{G} \text { is } r \text {-wise } t \text {-intersecting }\right\}, \\
\mathbf{G}_{j}(n, r, t) & =\left\{\mathscr{G} \subset 2^{[n]}: \mathscr{G} \subset \mathscr{G}^{\prime} \text { for some } \mathscr{G}^{\prime} \cong \mathscr{G}_{j}(n, r, t)\right\}, \\
\mathbf{X}^{i}(n, r, t) & =\mathbf{G}(n, r, t)-\bigcup_{0 \leq j \leq i} \mathbf{G}_{j}(n, r, t), \\
\mathbf{Y}^{i}(n, k, r, t) & =\left\{\mathscr{F} \subset\left(\begin{array}{c}
{[n]} \\
k
\end{array}\right): \mathscr{F} \in \mathbf{X}^{i}(n, r, t)\right\} .
\end{aligned}
$$

Finally let us define

$$
\begin{aligned}
m^{i}(n, k, r, t) & =\max \left\{|\mathscr{F}|: \mathscr{F} \in \mathbf{Y}^{i}(n, k, r, t)\right\}, \\
w^{i}(n, p, r, t) & =\max \left\{w_{p}(\mathscr{G}): \mathscr{G} \in \mathbf{X}^{i}(n, r, t)\right\} .
\end{aligned}
$$

Ahlswede and Khachatrian [1] determined $m^{0}(n, k, 2, t)$ completely, extending the earlier results by Hilton-Milner [18] and Frankl [11]. Brace and Daykin [4] determined $w^{0}(n, 1 / 2, r, 1)$ and Frankl determined $w^{0}(n, 1 / 2, r, t)$ for $r \geq 5$ and $1 \leq t \leq 2^{r}-r-1$; in both cases $\mathscr{G}_{1}(n, r, t)$ has the maximum $p$-weight. (But $\mathscr{G}_{1}$ is not always optimal for $w^{0}$, for example, we have $w^{0}(n, p, r, 1)>w_{p}\left(\mathscr{G}_{1}(n, r, 1)\right)$ if $p>\frac{1}{2}$ and $r \leq 5$, see [28].) More results for $m^{0}(n, k, r, t)$ with $k / n \approx 1 / 2$, and $w^{0}(n, p, r, t)$ with $p \approx 1 / 2$ are found in [17, 28, 29].

In this article we will introduce the random walk method originated by Frankl, which is a strong tool to investigate $w(n, p, r, t)$. In the next section, we explain the key idea of the method. In Section 3 we prepare some tools to apply the method. Then in Section 4 we illustrate the method by determining $w(n, 1 / 3,4,36)$, and a general setup to get $w(n, p, r, t)$ will be given in Section 5. In the last section we discuss how to derive $m(n, k, r, t)$ from $w(n, p, r, t)$ when $p \approx k / n$. As a consequence, we get the following result (see Theorem 10).

Theorem 1. Let $p_{0} \in(0,1)$ and $r, t, i \in \mathbb{N}$ be given. Suppose that $\max _{j}\left\{w_{p_{0}}\left(\mathscr{G}_{j}(n, r, t)\right)\right\}$ is attained by $j=i-1$ or $i$. Then (W) implies (M).

(W) There exist positive constants $\gamma_{0}, \varepsilon_{0}, n_{0}$ such that, for all $p$ with $\left|p-p_{0}\right|<\varepsilon_{0}$ and all $n$ with $n \geq n_{0}$, the following is true: If $\mathscr{G} \in \mathbf{X}^{i}(n, r, t)$ is shifted and $\cap \mathscr{G}=\emptyset$ then we have $w_{p}(\mathscr{G})<\left(1-\gamma_{0}\right) \max \left\{w_{p}\left(\mathscr{G}_{i-1}(n, r, t)\right), w_{p}\left(\mathscr{G}_{i}(n, r, t)\right)\right\}$.

(M) There exist positive constants $\varepsilon, n_{1}$ such that, for all $n>n_{1}$ and $k$ with $\left|\frac{k}{n}-p_{0}\right|<$ $\varepsilon$, we have (1) with equality holding only if $\mathscr{F}_{i-1}(n, k, r, t)$ or $\mathscr{F}_{i}(n, k, r, t)$ (up to isomorphism).

We can in fact show (W) in some particular choices of $p_{0}, r, t, i$ by the random walk method. As an example we verify (1) for $r \geq 4, t \leq\left(3^{r}-2 r-1\right) / 2, k / n \leq 1 / 3$, and $n$ large enough (Theorem 12). Although it is still beyond our reach to determine $m(n, k, r, t)$ and $w(n, p, r, t)$ completely, we hope that the strategy described in this article will provide a better understanding of multiply intersecting families. 


\section{THE RANDOM WALK METHOD}

In [10] Frankl found a way to connect the number of walks of certain types with an upper bound for the size of intersecting families. He then extended the idea to bound the size of 3-wise 2-intersecting families in [9], where the random walk method was explicitly used for the first time. One of the highlights of the method is [13], where he got many interesting results on multiply intersecting families, and most of them have no alternative proofs so far. A survey [12] by himself is highly recommended.

In this section we explain the key idea of the method. Let $p$ and $q$ be positive reals with $p+q=1$, and let $\alpha_{r, p} \in(p, 1)$ be the unique root of the equation $q x^{r}-x+p=0$. The random walk method is basically to use the following inequality:

$$
w(n, p, r, t) \leq \alpha_{r, p}^{t} \text {. }
$$

This inequality itself is not sharp, but we often get sharp upper bounds for the $p$-weight of intersecting families using (3) with some additional argument.

We outline how to get (3) here. (One can find the proof in [12] (for the case $p=1 / 2$ ) and we also include some more explanation about shifting technique etc. for convenience in the next section.) For $G \subset[n]$ we define the corresponding $n$-step walk on $\mathbb{Z}^{2}$, denoted by walk $(G)$, as follows. The walk is from $(0,0)$ to $(|G|, n-|G|)$, and the $i$-th step is one unit up ( $\uparrow$ ) if $i \in G$, or one unit to the right $(\rightarrow)$ if $i \notin G$. Let $\mathscr{G} \in \mathbf{G}(n, r, t)$. We can find a shifted $\mathscr{G}^{*} \in \mathbf{G}(n, r, t)$ with $w_{p}(\mathscr{G})=w_{p}\left(\mathscr{G}^{*}\right)$. Then, for each $G \in \mathscr{G}^{*}$, walk $(G)$ touches the line $L: y=(r-1) x+t$ (see Lemma 4). Thus we have $\mathscr{G}^{*} \subset \mathscr{W}_{n}$, where $\mathscr{W}_{n}=\{W \subset$ $[n]$ : walk $(W)$ touches $L\}$. We note that $\mathscr{W}_{n}$ is not necessarily $r$-wise $t$-intersecting.

Now consider the infinite random walk in $\mathbb{Z}^{2}$ starting from $(0,0)$, taking $\uparrow$ with probability $p$ and $\rightarrow$ with probability $q$ at each step independently. Suppose that $\mathscr{G}$ has the maximum $p$-weight. Then it follows that

$$
\begin{aligned}
w(n, p, r, t) & =\sum_{G \in \mathscr{G}} p^{|G|} q^{n-|G|} \leq \sum_{W \in \mathscr{W}_{n}} p^{|W|} q^{n-|W|} \leq \lim _{n \rightarrow \infty} \sum_{W \in \mathscr{W}_{n}} p^{|W|} q^{n-|W|} \\
& =\mathbf{P} \text { (the infinite random walk touches } L)=\alpha_{r, p}^{t} .
\end{aligned}
$$

The last equality (4) can be shown as follows. Let $X_{s}$ be the probability that the infinite random walk touches the line $y=(r-1) x+s$. After the first step, we are at $(1,0)$ with probability $p$, or at $(0,1)$ with probability $q$. Thus we have

$$
X_{t}=p X_{t-1}+q X_{t+r-1} \text {. }
$$

Let $a_{i}$ be the number of walks from $(0,0)$ to $A_{i}=(i,(r-1) i+t)$ which touch $L$ only at $A_{i}$. Then we have $X_{t}=\sum_{i \geq 0} a_{i} p^{(r-1) i+t} q^{i}$. To touch the line $L^{\prime}: y=(r-1) x+t+1$, we need to hit $L$ somewhere, say, at $A_{i}$ for the first time. Then the probability that we hit $L^{\prime}$ starting from $A_{i}$ is equal to $X_{1}$. Thus we have

$$
X_{t+1}=\sum_{i \geq 0}\left(a_{i} p^{(r-1) i+t} q^{i}\right) X_{1}=X_{t} X_{1}=X_{1}^{t+1} .
$$

By (5) and (6) we have $X_{1}=p+q X_{1}^{r}$. This equation has unique root $X_{1}=\alpha_{r, p}$ in $(0,1)$, and then (6) gives $X_{t}=\alpha_{r, p}^{t}$, which proves (4). One can also show that $a_{i}=\frac{t}{r i+t}\left(\begin{array}{c}r i+t \\ i\end{array}\right)$ and $\sum_{i \geq 0} a_{i} p^{(r-1) i+t} q^{i}=\alpha_{r, p}^{t}$ in a different way, see e.g., [22].

To consider the $k$-uniform version problem, let us review the very original idea of the method from [10]. Let $\mathscr{F} \subset\left(\begin{array}{c}{[n]} \\ k\end{array}\right)$ be 2 -wise $t$-intersecting. Then for every $F \in \mathscr{F}, \operatorname{walk}(F)$ 
is from $(0,0)$ to $(n-k, k)$, which touches the line $y=x+t$. The total number of walks with this property is, by the reflection principle, equal to the total number of walks from $(-t, t)$ to $(n-k, k)$, which is $\left(\begin{array}{c}n \\ k-t\end{array}\right)$. This gives $m(n, k, 2, t) \leq\left(\begin{array}{c}n \\ k-t\end{array}\right) \leq\left(\frac{k}{n-k}\right)^{t}\left(\begin{array}{l}n \\ k\end{array}\right)$. On the other hand, by setting $p=\frac{k}{n}$, we have $\alpha_{2, p}=\frac{p}{q}=\frac{k}{n-k}$, and $m(n, k, 2, t) \leq \alpha_{2, p}^{t}\left(\begin{array}{l}n \\ k\end{array}\right)$. This suggests the following $k$-uniform version of (3):

$$
m(n, k, r, t) \leq \alpha_{r, p}^{t}\left(\begin{array}{l}
n \\
k
\end{array}\right)
$$

where $p=\frac{k}{n}$. This is true if $p<\frac{r-1}{r+1}$ is fixed and $n$ is large enough, see [25]. We will discuss how to get $m(n, k, r, t)$ from $w(n, p, r, t)$ in the last section.

\section{TOOLS}

Let us introduce the shifting operation. For integers $1 \leq i<j \leq n$ and a family $\mathscr{G} \subset 2^{[n]}$, we define the $(i, j)$-shift $\sigma_{i j}$ as follows:

$$
\sigma_{i j}(\mathscr{G})=\left\{\sigma_{i j}(G): G \in \mathscr{G}\right\},
$$

where

$$
\sigma_{i j}(G)= \begin{cases}(G-\{j\}) \cup\{i\} & \text { if } i \notin G, j \in G,(G-\{j\}) \cup\{i\} \notin \mathscr{G}, \\ G & \text { otherwise. }\end{cases}
$$

This operation preserves $r$-wise $t$-intersecting property, namely, if $\mathscr{G}$ is $r$-wise $t$-intersecting, then so is $\sigma_{i j}(\mathscr{G})$. Note also that shifting does not change the $p$-weight, i.e., $w_{p}\left(\sigma_{i j}(\mathscr{G})\right)=$ $w_{p}(\mathscr{G})$.

A family $\mathscr{G} \subset 2^{[n]}$ is called shifted if $\sigma_{i j}(\mathscr{G})=\mathscr{G}$ for all $1 \leq i<j \leq n$, and $\mathscr{G}$ is called tame if it is shifted and $\bigcap \mathscr{G}=\emptyset$. Starting from a given $\mathscr{G}$ we can always get a shifted $\mathscr{G}^{\prime}$ by a finite sequence of shifting operations. To see this fact, let $s(\mathscr{G})=\sum\left\{\sum\{g: g \in G\}\right.$ : $G \in \mathscr{G}\} \in \mathbb{N}$ and observe $s\left(\sigma_{i j}(\mathscr{G})\right)<s(\mathscr{G})$ if $\sigma_{i j}(\mathscr{G}) \neq \mathscr{G}$.

Lemma 2. $\mathbf{X}^{0}(n, r, t) \subset \mathbf{X}^{0}(n, r-1, t+1)$ and $w^{0}(n, p, r, t) \leq w^{0}(n, p, r-1, t+1)$.

Proof. Let $\mathscr{G} \in \mathbf{X}^{0}(n, r, t)$. Then clearly we have $\mathscr{G} \notin \mathbf{G}_{0}(n, r-1, t+1)$. Thus it suffices to show that $\mathscr{G} \in \mathbf{G}(n, r-1, t+1)$. If it is not, then we can find $G_{1}, \ldots, G_{r-1} \in \mathscr{G}$ such that $\left|G_{1} \cap \cdots \cap G_{r-1}\right|=t$. But $\mathscr{G}$ is $r$-wise $t$-intersecting and so every $G \in \mathscr{G}$ must contain $G_{1} \cap \cdots \cap G_{r-1}$. This means $\mathscr{G} \notin \mathbf{X}^{0}(n, r, t)$, a contradiction.

Lemma 3. If $\mathscr{G} \in \mathbf{X}^{0}(n, r, t)$ has maximum $p$-weight then we can find a tame $\mathscr{G}^{\prime} \in \mathbf{X}^{0}(n, r, t)$ with $w_{p}\left(\mathscr{G}^{\prime}\right)=w_{p}(\mathscr{G})$.

Proof. If $\mathscr{G} \in \mathbf{X}^{0}(n, r, t)$ then $\mathscr{G} \in \mathbf{X}^{0}(n, r-1, t+1)$ by Lemma 2 . We apply shifting operations to $\mathscr{G}$ to get a shifted family $\mathscr{G}^{\prime} \in \mathbf{G}(n, r, t) \subset \mathbf{G}(n, r-1, t+1)$.

We have to show that $\bigcap \mathscr{G}^{\prime}=\emptyset$. Otherwise we may assume that $1 \in \bigcap \mathscr{G}^{\prime}$ and $H=$ $[2, n] \notin \mathscr{G}^{\prime}$. Since $\mathscr{G}^{\prime}$ is $p$-weight maximum we can find $G_{1}, \ldots, G_{r-1} \in \mathscr{G}^{\prime}$ such that $\mid G_{1} \cap$ $\cdots \cap G_{r-1} \cap H \mid<t$. Then we have $\left|G_{1} \cap \cdots \cap G_{r-1}\right|<t+1$, which is a contradiction.

Lemma 4. Let $\mathscr{G} \in \mathbf{G}(n, r, t)$ be shifted. Then walk $(G)$ touches the line $L: y=(r-1) x+t$ for all $G \in \mathscr{G}$.

Proof. Let $H=[n]-\{t, t+r, t+2 r, t+3 r, \ldots\}$. Then walk $(H)$ does not touch $L$. Moreover this walk is the maximal one with this property. Namely, if walk $(F)$ does not touch $L$, then we can find $F^{\prime} \supset F$ such that $H$ is obtained from $F^{\prime}$ by a sequence of shifting operations. 
Let $\mathscr{G} \in \mathbf{G}(n, r, t)$. Suppose that we have some $G \in \mathscr{G}$ such that walk $(G)$ does not touch $L$. We may assume that $\mathscr{G}$ is size maximal, and so $G=H$. For $1 \leq i<r$, let $H_{i}=[n]-\{t+i, t+r+i, t+2 r+i, t+3 r+i, \ldots\}$. We get $H_{i}$ from $H$ by shifting. Since $\mathscr{G}$ is shifted we have $H, H_{1}, \ldots, H_{r-1} \in \mathscr{G}$ and $H \cap H_{1} \cap \cdots \cap H_{r-1}=[t-1]$, which is a contradiction.

Lemma 5 ([28]). Let $p, r, t_{0}, c$ be fixed constants, and let $\alpha \in(p, 1)$ be the root of the equation $q x^{r}-x+p=0$. Suppose that $w\left(n, p, r, t_{0}\right) \leq c$ holds for all $n \geq t_{0}$. Then we have $w(n, p, r, t) \leq c \alpha^{t-t_{0}}$ for all $t \geq t_{0}$ and $n \geq t$.

Proof. If $\mathscr{G} \subset 2^{[n]}$ is trivial $r$-wise $t_{0}$-intersecting, i.e., $|\cap \mathscr{G}| \geq t_{0}$, then we have $\mathscr{G} \subset\{G \subset$ $\left.[n]:\left[t_{0}\right] \subset G\right\}$ and $w_{p}(\mathscr{G}) \leq p^{t_{0}}$. Thus we may assume that $c \geq p^{t_{0}}$. Note also that $p<\alpha$.

We prove the result by double induction on $s=n-t$ and $t$. One of the initial steps for $t=t_{0}$ follows from our assumption. For the other initial step for $s$, we prove the result for the cases $0 \leq s \leq r-1$, or equivalently, $t \leq n \leq t+r-1$. Suppose that $\mathscr{G} \subset 2^{[n]}$ satisfies $w_{p}(\mathscr{G})=w(n, p, r, t)$. We may assume that $\mathscr{G}$ is shifted and size maximal. If $\mathscr{G}$ is trivial, i.e., $|\cap \mathscr{G}| \geq t$, then we have $w_{p}(\mathscr{G}) \leq p^{t}=p^{t_{0}} p^{t-t_{0}}<c \alpha^{t-t_{0}}$ and we are done. Otherwise we have $G \in \mathscr{G}$ such that $[t] \not \subset G$, and we may assume that $G_{t}=[n]-\{t\} \in \mathscr{G}$ because $\mathscr{G}$ is shifted and maximal. Then again by the shiftedness we have $G_{i}=[n]-\{i\} \in \mathscr{G}$ for all $t \leq i \leq n$. This implies $\left|\bigcap_{i=t}^{n} G_{i}\right|=t-1$. But this is impossible because $\mathscr{G}$ is $r$-wise $t$-intersecting and $n-t+1 \leq r$.

Next we show the induction step. Let $s \geq r$ and $t>t_{0}$. We show the case $(s, t)$. We assume that the result holds for $\{(s, b): b<t\} \cup\left\{(a, b): a<s, b \geq t_{0}\right\}$. In particular, we can apply induction hypothesis to the case $(s, t-1)$ and $(s-r, t+r-1)$.

Let $\mathscr{G} \subset 2^{[n]}$ be $r$-wise $t$-intersecting. Define $\mathscr{G}_{1}, \mathscr{G}_{\overline{1}} \subset 2^{[2, n]}$ as follows:

$$
\mathscr{G}_{1}=\{G-\{1\}: 1 \in G \in \mathscr{G}\}, \quad \mathscr{G}_{\overline{1}}=\{G: 1 \notin G \in \mathscr{G}\} .
$$

Then $\mathscr{G}_{1}$ is clearly $r$-wise $(t-1)$-intersecting. On the other hand, $\mathscr{G}_{\overline{1}}$ is $r$-wise $(t+r-1)$ intersecting. To see this fact suppose, on the contrary, that there exist $G_{2} \ldots G_{r+1} \in \mathscr{G}_{\overline{1}}$ such that $\bigcap_{i=2}^{r+1} G_{i}=[2, t+r-1]$. By the shiftedness we have $G_{i}^{\prime}=\{1\} \cup\left(G_{i}-\{i\}\right) \in \mathscr{G}$ for all $2 \leq i \leq r+1$. But then we have $\bigcap_{i=2}^{r+1} G_{i}^{\prime}=[t+r-1]-[2, r+1]$, which contradicts $r$-wise $t$-intersecting property of $\mathscr{G}$.

Note that $s$ for $\mathscr{G}_{1}$ is $(n-1)-(t-1)=s$ and $s$ for $\mathscr{G}_{\overline{1}}$ is $(n-1)-(t+r-1)=s-r$. Therefore using the induction hypothesis, we have

$$
\begin{aligned}
w_{p}(\mathscr{G}) & =p w_{p}\left(\mathscr{G}_{1}:[2, n]\right)+q w_{p}\left(\mathscr{G}_{\overline{1}}:[2, n]\right) \leq p c \alpha^{t-t_{0}-1}+q c \alpha^{t+r-t_{0}-1} \\
& =c \alpha^{t-t_{0}-1}\left(p+q \alpha^{r}\right)=c \alpha^{t-t_{0}} \cdot \square
\end{aligned}
$$

Lemma 6. For any $i \geq 0$ we have $w^{i}(n+1, p, r, t) \geq w^{i}(n, p, r, t)$.

Proof. Choose $\mathscr{G} \in \mathbf{X}^{i}(n, r, t)$ with $w_{p}(\mathscr{G})=w^{i}(n, p, r, t)$. Then $\mathscr{G}^{\prime}:=\mathscr{G} \cup\{G \cup\{n+1\}$ : $G \in \mathscr{G}\} \in \mathbf{X}^{i}(n+1, r, t)$ and $w_{p}\left(\mathscr{G}^{\prime}:[n+1]\right)=w_{p}(\mathscr{G}:[n])(q+p)=w^{i}(n, p, r, t)$, which means $w^{i}(n+1, p, r, t) \geq w^{i}(n, p, r, t)$.

\section{AN EXAMPLE}

As a toy example, we consider the case $r=4$ and $t=36$. Let $p \in(0,1)$ and $q=1-p$, and set $\mathscr{G}_{j}=\mathscr{G}_{j}(n, 4,36)$. Simple computation shows that $w_{p}\left(\mathscr{G}_{0}\right) \geq w_{p}\left(\mathscr{G}_{1}\right)$ iff $p \leq 1 / 3$. 
To give a feel of the random walk method, we will show that

$$
w(n, p, 4,36)=w_{p}\left(\mathscr{G}_{0}\right)=p^{36}
$$

for all $n \geq 40$ and $p \leq 1 / 3$.

Clearly we have $w(n, p, 4,36) \leq w(n, p, 2,36)$, and the Ahlswede-Khachatrian result [3] already shows (7) for $p \leq 1 /(t+1)=1 / 37$. We can easily improve this upper bound for $p$ using (3). Suppose that $\mathscr{G} \in \mathbf{G}(n, 4,36)$. If $\mathscr{G} \in \mathbf{G}_{0}(n, 4,36)$ then we have $w_{p}(\mathscr{G}) \leq p^{36}$. Otherwise we have $\mathscr{G} \in \mathbf{X}^{0}(n, 4,36) \subset \mathbf{X}^{0}(n, 3,37)$ by Lemma 2 . Now by (3) we have $w_{p}(\mathscr{G}) \leq \alpha_{3, p}^{37}$. Then we find that $\alpha_{3, p}^{37}<p^{36}$ if $p \leq 1 / 5$. In this way we get (7) for $p \leq 1 / 5$.

To get (7) for $p \leq 1 / 3$, we will prove the following slightly stronger inequality, that is,

$$
w^{1}(n, p, 4,36)<0.9999 \max \left\{w_{p}\left(\mathscr{G}_{0}\right), w_{p}\left(\mathscr{G}_{1}\right)\right\}
$$

for all $n \geq 40$ and $p \leq 0.34$. This gives $w(n, p, 4,36)=\max \left\{w_{p}\left(\mathscr{G}_{0}\right), w_{p}\left(\mathscr{G}_{1}\right)\right\}$ for $p \leq 0.34$, and in particular this implies (7) for $p \leq 1 / 3$.

Choose $\mathscr{G} \in \mathbf{X}^{1}(n, 4,36)$ with the maximum $p$-weight, and choose a tame $\mathscr{G}^{*} \in \mathbf{X}^{0}(n, 4,36)$ with $w_{p}(\mathscr{G})=w_{p}\left(\mathscr{G}^{*}\right)$ by Lemma 3 . We will show the following.

(i) If $\mathscr{G}^{*} \not \subset \mathscr{G}_{1}$ then $w_{p}\left(\mathscr{G}^{*}\right)<0.99 w_{p}\left(\mathscr{G}_{0}\right)$ for $p \leq 0.34$.

(ii) If $\mathscr{G}^{*} \subset \mathscr{G}_{1}$ then $w_{p}\left(\mathscr{G}^{*}\right)<0.9999 w_{p}\left(\mathscr{G}_{1}\right)$ for $p \leq 0.34$.

We can show (ii) in a more general setting as we will see in the next section. Here we show (i). So we assume that $\mathscr{G}^{*} \not \subset \mathscr{G}_{1}$ and rename it $\mathscr{G}$.

Let $s=\max \{j: \mathscr{G} \in \mathbf{G}(n, 3, j)\}$. By Lemma 2 we have $s \geq 37$. If $s \geq 40$ then by (3) we have

$$
w_{p}(\mathscr{G}) \leq w(n, p, 3,40) \leq \alpha_{3, p}^{40}<0.99 p^{36}
$$

for $p \leq 0.34$. Thus we may assume that $37 \leq s \leq 39$. After [13] let

$$
h=\min \{j:|G \cap[36+j]| \geq 36 \text { for all } G \in \mathscr{G}\} .
$$

This is the maximum size of "holes" in $[36+h]$.

Claim 1. $1 \leq h \leq s-36 \leq 3$.

Proof. Since $\mathscr{G} \in \mathbf{X}^{0}(n, 4,36)$, we have $h \geq 1$. By the definition of $s$ and the shiftedness of $\mathscr{G}$, we have $G_{1}, G_{2}, G_{3} \in \mathscr{G}$ such that $G_{1} \cap G_{2} \cap G_{3}=[s]$. Since $\mathscr{G} \in \mathbf{G}(n, 4,36)$ it follows that $|G \cap[s]| \geq 36$ for all $G \in \mathscr{G}$, namely, $36+h \leq s$.

Let $b=36+(h-1)=35+h$ and let $T_{i}=[b+1-i, b]$ be the right-most $i$-set in $[b]$ $\left(T_{0}=\emptyset\right)$. For $A \subset[b]$ let

$$
\mathscr{G}(A)=\{G \cap[b+1, n]: G \in \mathscr{G},[b] \backslash G=A\} .
$$

Since $\mathscr{G}$ is shifted, we have $\mathscr{G}(A) \subset \mathscr{G}\left(T_{i}\right)$ for all $A \in\left(\begin{array}{c}{[b]} \\ i\end{array}\right)$, and thus we have

$$
w_{p}(\mathscr{G}) \leq \sum_{i=0}^{h}\left(\begin{array}{l}
b \\
i
\end{array}\right) p^{b-i} q^{i} w_{p}\left(\mathscr{G}\left(T_{i}\right):[b+1, n]\right) .
$$

To bound $w_{p}\left(\mathscr{G}\left(T_{i}\right):[b+1, n]\right)$ we use the fact that $\mathscr{G}\left(T_{i}\right)$ is highly-intersecting as we see below.

Claim 2. For $0 \leq i<h$ we have $\mathscr{G}\left(T_{i}\right) \in \mathbf{G}(n, 3,3 i+1)$. 
Proof. Suppose that $\mathscr{G}\left(T_{i}\right) \notin \mathbf{G}(n, 3,3 i+1)$. Then we can find $G_{1}, G_{2}, G_{3} \in \mathscr{G}\left(T_{i}\right)$ such that $\left|G_{1} \cap G_{2} \cap G_{3}\right| \leq 3 i$. Since $\mathscr{G}$ is shifted, we may assume that $G_{1} \cap G_{2} \cap G_{3} \subset[b+1, b+3 i]$. For $1 \leq \ell \leq 3$, by shifting $\left(G_{\ell} \cup[b]\right)-T_{i} \in \mathscr{G}$, we get $G_{\ell}^{\prime}:=\left(G_{\ell} \cup[b]\right)-[b+1+(\ell-$ $1) i, b+\ell i] \in \mathscr{G}$. By the definition of $h$ we have some $H \in \mathscr{G}$ such that $|H \cap[b]|<36$ and due to the shiftedness of $\mathscr{G}$ we may assume that $H=[n]-[36, b]$. Then we have $G_{1}^{\prime} \cap G_{2}^{\prime} \cap G_{3}^{\prime} \cap H=[35]$, which contradicts the fact $\mathscr{G} \in \mathbf{G}(n, 4,36)$.

Claim 3. If $\mathscr{G} \not \subset \mathscr{G}_{h}$ then $\mathscr{G}\left(T_{h}\right) \in \mathbf{G}(n, 3,3 h+2)$.

Proof. Suppose that $\mathscr{G}\left(T_{h}\right) \notin \mathbf{G}(n, 3, w)$, where $w=3 h+2$. Then we can find $G_{1}, G_{2}, G_{3} \in$ $\mathscr{G}\left(T_{h}\right)$ such that $G_{1} \cap G_{2} \cap G_{3} \subset[b+1, b+w-1]$. By shifting $\left(G_{\ell} \cup[b]\right)-T_{h} \in \mathscr{G}$ we get $G_{\ell}^{\prime}:=\left(G_{\ell} \cup[b]\right)-[36+(\ell-1) h, 35+\ell h] \in \mathscr{G}$ for $1 \leq \ell \leq 3$. Since $\mathscr{G} \not \subset \mathscr{G}_{h}$ we have $G_{4}^{\prime}:=[n]-[36+3 h, 36+4 h] \in \mathscr{G}$. Then we have $\left|G_{1}^{\prime} \cap \cdots \cap G_{4}^{\prime}\right|<36$, a contradiction.

We may assume that $\mathscr{G} \not \subset \mathscr{G}_{h}$ for $1 \leq h \leq 3$. In fact, we have already assumed $\mathscr{G} \not \subset \mathscr{G}_{1}$, and we have $w_{p}\left(\mathscr{G}_{i}\right)<0.99 \max \left\{w_{p}\left(\mathscr{G}_{0}\right), w_{p}\left(\mathscr{G}_{1}\right)\right\}$ for $i=2,3$ and $p \leq 0.34$.

First we consider the case $h=1$. In this case, by Claim 2 we have $\mathscr{G}\left(T_{0}\right) \in \mathbf{G}(n, 3,1)$. Since $\mathscr{G} \not \subset \mathscr{G}_{1}$ it follows from Claim 3 that $\mathscr{G}\left(T_{1}\right) \in \mathbf{G}(n, 3,5)$. Thus (3) gives $w_{p}\left(\mathscr{G}\left(T_{0}\right)\right.$ : $[b+1, n]) \leq \alpha_{3, p}$ and $w_{p}\left(\mathscr{G}\left(T_{1}\right):[b+1, n]\right) \leq \alpha_{3, p}^{5}$. Finally by (10) we have

$$
w_{p}(\mathscr{G}) \leq p^{36} \alpha_{3, p}+36 p^{35} q \alpha_{3, p}^{5}<0.99 p^{36}
$$

for $p \leq 0.34$.

Next we consider the case $h=2$. In this case, Claim 2 gives $\mathscr{G}\left(T_{0}\right) \in \mathbf{G}(n, 3,1)$ and $\mathscr{G}\left(T_{1}\right) \in \mathbf{G}(n, 3,4)$, and Claim 3 gives $\mathscr{G}\left(T_{2}\right) \in \mathbf{G}(n, 3,8)$. Thus (3) and (10) imply

$$
w_{p}(\mathscr{G}) \leq p^{37} \alpha_{3, p}+37 p^{36} q \alpha_{3, p}^{4}+\left(\begin{array}{c}
37 \\
2
\end{array}\right) p^{35} q^{2} \alpha_{3, p}^{8}<0.99 p^{36} .
$$

Similarly, in the case $h=3$, we have

$$
w_{p}(\mathscr{G}) \leq p^{38} \alpha_{3, p}+38 p^{37} q \alpha_{3, p}^{4}+\left(\begin{array}{c}
38 \\
2
\end{array}\right) p^{36} q^{2} \alpha_{3, p}^{7}+\left(\begin{array}{c}
38 \\
3
\end{array}\right) p^{35} q^{3} \alpha_{3, p}^{11}<0.99 p^{36} .
$$

This completes the proof of (i).

If we have more information about $w(n, p, 3, *)$ then we get simpler proof. For example, using a result in [27] we have $w(n, p, 3,8) \leq p^{8}$ for $p \leq 0.34$. This together with Lemma 5 gives

$$
w(n, p, 3,39) \leq p^{8} \alpha_{3, p}^{31}<0.99 p^{36} .
$$

By replacing (9) with the above estimation, we can conclude that $37 \leq s \leq 38$ and so $1 \leq h \leq 2$. This means we do not have to deal with (11).

\section{A GenERAL SETUP}

Let $n, p, r, t$ be fixed and let $\mathscr{G}_{i}=\mathscr{G}_{i}(n, r, t)$. Suppose that $\max \left\{w_{p}\left(\mathscr{G}_{i-1}\right), w_{p}\left(\mathscr{G}_{i}\right)\right\}>$ $w_{p}\left(\mathscr{G}_{j}\right)$ for all $j \notin\{i-1, i\}$, and consider the situation that we are trying to show

$$
w(n, p, r, t)=\max \left\{w_{p}\left(\mathscr{G}_{i-1}\right), w_{p}\left(\mathscr{G}_{i}\right)\right\},
$$

with equality holding only if $\mathscr{G} \cong \mathscr{G}_{i-1}$ or $\mathscr{G}_{i}$. If $\mathscr{G} \notin \mathbf{X}^{i}(n, r, t)$ then there is nothing to show. So suppose that $\mathscr{G} \in \mathbf{X}^{i}(n, r, t)$ and we want to show that $w_{p}(\mathscr{G})$ is much less than $\max \left\{w_{p}\left(\mathscr{G}_{i-1}\right), w_{p}\left(\mathscr{G}_{i}\right)\right\}$. Let $\mathscr{G}^{*}$ be a tame family obtained from $\mathscr{G}$ by shifting. Then we have two cases: 
(a) $\mathscr{G}^{*} \not \subset \mathscr{G}_{j}(n, r, t)$ for all $0 \leq j \leq i$. This is the essential case we need to estimate $w_{p}\left(\mathscr{G}^{*}\right)$ by the random walk method. To use the method, it is important that $\mathscr{G}^{*}$ is shifted. We saw an example in this case in the previous section.

(b) $\mathscr{G}^{*} \subset \mathscr{G}_{j}(n, r, t)$ for some $0 \leq j \leq i$. In this case, we will see that $w_{p}\left(\mathscr{G}^{*}\right)$ cannot be large by Theorem 7 below.

Consequently, to get (12) with the uniqueness of the optimal configuration, it is enough to consider a tame $\mathscr{G} \in \mathbf{X}^{i}(n, r, t)$ from the beginning.

Theorem 7. Let $r, t$ and $i$ be positive integers with $r \geq 4$, and let $p \in\left(0, \frac{r-3}{r-2}\right]$. Then there exists $\gamma>0$ such that for all $n \geq t+r$ the following is true.

Let $\mathscr{G} \in \mathbf{X}^{i}(n, r, t)$, and let $\mathscr{G}^{*} \in \mathbf{X}^{0}(n, r, t)$ be a tame family obtained from $\mathscr{G}$ by shifting. If $\mathscr{G}^{*} \subset \mathscr{G}_{i}(n, r, t)$ then

$$
w_{p}(\mathscr{G})<(1-\gamma) w_{p}\left(\mathscr{G}_{i}(n, r, t)\right) \text {. }
$$

Proof. Set $\mathscr{G}_{i}=\mathscr{G}_{i}(n, r, t)$. Note that $\mathscr{G}$ is not necessarily shifted. Since $\mathscr{G}^{*} \subset \mathscr{G}_{i}$, we may assume (by renaming the starting family if necessary) that $\mathscr{G}^{*}=\sigma_{x y}(\mathscr{G}) \subset \mathscr{G}_{i}$, where $x=t+r i, y=x+1$. We note that $|[x] \backslash G| \leq i+1$ for all $G \in \mathscr{G}$. Moreover if $|[x] \backslash G|=i+1$ then $G \cap\{x, y\}=\{y\}$ and $(G-\{y\}) \cup\{x\} \notin \mathscr{G}$.

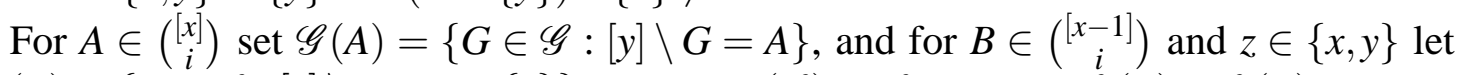
$\mathscr{G}_{z}(B)=\{G \in \mathscr{G}:[y] \backslash G=B \cup\{z\}\}$. Since $\sigma_{x y}(\mathscr{G}) \subset \mathscr{G}_{i}$ we have $\mathscr{G}_{x}(B) \cap \mathscr{G}_{y}(B)=\emptyset$ and so $w_{p}\left(\mathscr{G}_{x}(B)\right)+w_{p}\left(\mathscr{G}_{y}(B)\right) \leq p^{x-i} q^{i+1}$. Set $\mathscr{G}^{\prime}=\{G \in \mathscr{G}:|[x] \backslash G|<i\}, \mathscr{G}^{\prime \prime}=\{G \in \mathscr{G}$ : $|[x-1] \backslash G|=i-1, G \cap\{x, y\}=\emptyset\}$ and let $e=\min \left\{w_{p}(\mathscr{G}(A)): A \in\left(\begin{array}{c}{[x]} \\ i\end{array}\right)\right\}$. Then we have

$$
\begin{aligned}
w_{p}(\mathscr{G}) & =\sum_{A \in\left(\begin{array}{c}
{[x]} \\
i
\end{array}\right)} w_{p}(\mathscr{G}(A))+\sum_{B \in\left(\begin{array}{c}
{[x-1]} \\
i
\end{array}\right)}\left(w_{p}\left(\mathscr{G}_{x}(B)\right)+w_{p}\left(\mathscr{G}_{y}(B)\right)\right)+w_{p}\left(\mathscr{G}^{\prime}\right)+w_{p}\left(\mathscr{G}^{\prime \prime}\right) \\
& \leq e+\left(\left(\begin{array}{c}
x \\
i
\end{array}\right)-1\right) p^{x-i+1} q^{i}+\left(\begin{array}{c}
x-1 \\
i
\end{array}\right) p^{x-i} q^{i+1}+\sum_{j=0}^{i-1}\left(\begin{array}{c}
x \\
j
\end{array}\right) p^{x-j} q^{j}+\left(\begin{array}{c}
x-1 \\
i-1
\end{array}\right) p^{x-i} q^{i+1} \\
& =e+(\eta-1) p^{x-i+1} q^{i},
\end{aligned}
$$

where $\eta=\sum_{j=0}^{i}\left(\begin{array}{l}x \\ j\end{array}\right) p^{i-j-1} q^{-i+j}$. Note that $e \leq p^{x-i+1} q^{i}$, and (14) coincides $w_{p}\left(\mathscr{G}_{i}\right)=$ $\eta p^{x-i+1} q^{i}$ iff $e=p^{x-i+1} q^{i}$. If there is some $B \in\left(\begin{array}{c}{\left[\begin{array}{c}x-1 \\ i\end{array}\right)} \\ )\end{array}\right)$ such that $\mathscr{G}_{x}(B) \cup \mathscr{G}_{y}(B)=\emptyset$, then by (13) we get $w_{p}(\mathscr{G}) \leq w_{p}\left(\mathscr{G}_{i}\right)-p^{x-i} q^{i+1}=(1-q /(\eta p)) w_{p}\left(\mathscr{G}_{i}\right)$, and we are done. Thus we may assume that

$$
\mathscr{G}_{x}(B) \cup \mathscr{G}_{y}(B) \neq \emptyset \text { for all } B \in\left(\begin{array}{c}
{[x-1]} \\
i
\end{array}\right) .
$$

To prove $w_{p}(\mathscr{G})<(1-\gamma) w_{p}\left(\mathscr{G}_{i}\right)$ by contradiction, let us assume that for any $\gamma>0$ and any $n_{0}$ there is some $n>n_{0}$ such that

$$
w_{p}(\mathscr{G})>(1-\gamma) w_{p}\left(\mathscr{G}_{i}\right)=(1-\gamma) \eta p^{x-i+1} q^{i} .
$$

By (14) and (16) we have $e>(1-\gamma \eta) p^{x-i+1} q^{i}$. This means, letting $\mathscr{H}(A)=\{G \backslash[y]$ : $G \in \mathscr{G}(A)\}$ and $Y=[y+1, n]$, we have $w_{p}(\mathscr{H}(A): Y)>1-\gamma \eta$, namely,

$$
w_{p}\left(2^{Y}-\mathscr{H}(A): Y\right)>\gamma \eta \text { for all } A \in\left(\begin{array}{c}
{[x]} \\
i
\end{array}\right) .
$$

Since $\mathscr{G} \in \mathbf{X}^{i}(n, r, t)$ both $\bigcup_{B \in\left(\begin{array}{c}{[x-1]} \\ i\end{array}\right)} \mathscr{G}_{x}(B)$ and $\bigcup_{B \in\left(\begin{array}{c}x-1 \\ i\end{array}\right)} \mathscr{G}_{y}(B)$ are non-empty. Using this with (15), we can choose $G \in \mathscr{G}_{x}(B)$ and $G^{\prime} \in \mathscr{G}_{y}\left(B^{\prime}\right)$ with $B, B^{\prime} \in\left(\begin{array}{c}{[x-1]} \\ i\end{array}\right)$ and $B \cap B^{\prime}=\emptyset$. 
Let $L=[x-1]-\left(B \cup B^{\prime}\right)$ and $\mathscr{H}^{*}=\bigcap_{A \in\left(\begin{array}{c}L \\ i\end{array}\right)} \mathscr{H}(A)$. Then by (17) we have

$$
\begin{aligned}
w_{p}\left(\mathscr{H}^{*}: Y\right) & =1-w_{p}\left(2^{Y}-\mathscr{H}^{*}: Y\right)=1-w_{p}\left(\cup_{A \in\left(\begin{array}{c}
L \\
i
\end{array}\right)}\left(2^{Y}-\mathscr{H}(A)\right): Y\right) \\
& \geq 1-\sum_{A \in\left(\begin{array}{c}
L \\
i
\end{array}\right)} w_{p}\left(2^{Y}-\mathscr{H}(A): Y\right)>1-\left(\begin{array}{c}
|L| \\
i
\end{array}\right) \gamma \eta .
\end{aligned}
$$

If $\mathscr{H}^{*} \subset 2^{Y}$ is not $(r-2)$-wise 1 -intersecting, then we can find $H_{1}, \ldots H_{r-2} \in \mathscr{H}^{*}$ such that $H_{1} \cap \cdots \cap H_{r-2}=\emptyset$. Choose disjoint $i$-sets $B_{\ell} \subset L, 1 \leq \ell \leq r-2$, and set $G_{\ell}:=([y]-$ $\left.B_{\ell}\right) \cup H_{\ell} \in \mathscr{G}$. Then we have $\left|G_{1} \cap \cdots \cap G_{r-2} \cap G \cap G^{\prime}\right|=t-1$, which contradicts the $r$-wise $t$-intersecting property of $\mathscr{G}$. Thus $\mathscr{H}^{*}$ is $(r-2)$-wise 1 -intersecting and $w_{p}\left(\mathscr{H}^{*}: Y\right) \leq p$ by (2). (We need $r \geq 4$ and $p \leq \frac{r-3}{r-2}$ here.) But this contradicts (18) because we can choose $\gamma$ so small that $p \ll 1-\left(\begin{array}{c}|L| \\ i\end{array}\right) \gamma \eta$.

This theorem implies (ii) of the previous section by taking $\gamma=0.0001$. In fact we have $q /(\eta p)>\gamma$ and $p \leq 1-\left(\begin{array}{c}|L| \\ i\end{array}\right) \gamma \eta=1-37 \gamma\left(\frac{1}{q}+\frac{40}{p}\right)$ for $p \leq 0.34$. Consequently we have proved (8). It is an easy exercise to get

$$
w^{1}(n, p, 4, t)<(1-\gamma) \max \left\{w_{p}\left(\mathscr{G}_{0}(n, 4, t)\right), w_{p}\left(\mathscr{G}_{1}(n, 4, t)\right)\right\}
$$

for all $n \geq 40,1 \leq t \leq 36$ and $p \leq 0.34$, where $\gamma>0$ is an absolute constant. Then using induction on $r$ with more careful analysis (but very much in the same way we did for the case $r=4$ and $t=36$ ) one can show the following.

Theorem 8. For all $r \geq 4$ there exist positive constants $\varepsilon, \gamma$ such that

$$
w^{1}(n, p, r, t)<(1-\gamma) \max \left\{w_{p}\left(\mathscr{G}_{0}(n, r, t)\right), w_{p}\left(\mathscr{G}_{1}(n, r, t)\right)\right\}
$$

holds for all $n \geq t+r, 1 \leq t \leq\left(3^{r}-2 r-1\right) / 2$ and $p \leq \frac{1}{3}+\varepsilon$.

We note that $w_{p}\left(\mathscr{G}_{0}(n, r, t)\right)=w_{p}\left(\mathscr{G}_{1}(n, r, t)\right)$ if $p=1 / 3$ and $t=\left(3^{r}-2 r-1\right) / 2$. As a corollary we get the following.

Corollary 9. For all $r \geq 4, n \geq t+r, 1 \leq t \leq\left(3^{r}-2 r-1\right) / 2$ and $p \leq 1 / 3$ we have

$$
w(n, p, r, t)=w_{p}\left(\mathscr{G}_{0}(n, r, t)\right)=p^{t} .
$$

Moreover if $t=\left(3^{r}-2 r-1\right) / 2$ and $p=1 / 3$ then $\mathscr{G}_{0}(n, r, t)$ and $\mathscr{G}_{1}(n, r, t)$ are the only optimal configurations (up to isomorphism). Otherwise $\mathscr{G}_{0}(n, r, t)$ is the only optimal configuration (up to isomorphism).

\section{FROM $p$-WEIGHT VERSION TO $k$-UNIFORM VERSION}

In this section, we show that a $k$-uniform version problem for $m(n, k, r, t)$ can be reduced to a $p$-weight version problem for $w(n, p, r, t)$ when $k / n \approx p$ (Theorems 10 and 11 ). Using these results, we will get a $k$-uniform version (Theorem 12) corresponding to Theorem 8. Theorem 1 in the introduction is an immediate consequence of the following result.

Theorem 10. Let $p_{0} \in(0,1)$ and $r, t, i \in \mathbb{N}$ be given. Then (W) implies (M).

(W) There exist positive constants $\gamma_{0}, \varepsilon_{0}, n_{0}$ such that

$$
w^{i}(n, p, r, t)<\left(1-\gamma_{0}\right) \max \left\{w_{p}\left(\mathscr{G}_{i-1}(n, r, t)\right), w_{p}\left(\mathscr{G}_{i}(n, r, t)\right)\right\}
$$

holds for all $p$ with $\left|p-p_{0}\right|<\varepsilon_{0}$ and all $n$ with $n \geq n_{0}$. 
(M) There exist positive constants $\gamma, \varepsilon, n_{1}$ such that

$$
m^{i}(n, k, r, t)<(1-\gamma) \max \left\{\left|\mathscr{F}_{i-1}(n, k, r, t)\right|,\left|\mathscr{F}_{i}(n, k, r, t)\right|\right\}
$$

holds for all $n>n_{1}$ and $k$ with $\left|\frac{k}{n}-p_{0}\right|<\varepsilon$. (We can choose $\varepsilon=\frac{\varepsilon_{0}}{2}, \gamma=\frac{\gamma_{0}}{4}$.)

For reals $0<b<a$ we write $a \pm b$ to mean the open interval $(a-b, a+b)$, and for $n \in \mathbb{N}, n(a \pm b)$ means $((a-b) n,(a+b) n) \cap \mathbb{N}$.

Proof. Assuming the negation of (M), we will construct a counterexample to (W).

For fixed $r$ and $t$ we note that

$$
f(p):=\max \left\{w_{p}\left(\mathscr{G}_{i-1}(n, r, t)\right), w_{p}\left(\mathscr{G}_{i}(n, r, t)\right)\right\}
$$

is a uniformly continuous function of $p$ on $p_{0} \pm \varepsilon_{0}$. Let $\varepsilon=\frac{\varepsilon_{0}}{2}, \gamma=\frac{\gamma_{0}}{4}$, and $I=p_{0} \pm \varepsilon$.

Choose $\varepsilon_{1} \ll \varepsilon$ so that

$$
(1-3 \gamma) f(p)>(1-4 \gamma) f(p+\delta)
$$

holds for all $p \in I$ and all $0<\delta \leq \varepsilon_{1}$. Choose $n_{2}$ so that

$$
\sum_{j \in J}\left(\begin{array}{l}
n \\
j
\end{array}\right) p_{1}^{j}\left(1-p_{1}\right)^{n-j}>(1-3 \gamma) /(1-2 \gamma)
$$

holds for all $n>n_{2}$ and all $p_{1} \in I_{0}:=p_{0} \pm \frac{3 \varepsilon}{2}$, where $J=n\left(p_{1} \pm \varepsilon_{1}\right)$. Choose $n_{3}$ so that

$$
(1-\gamma) \max \left\{\left|\mathscr{F}_{i-1}(n, k, r, t)\right|,\left|\mathscr{F}_{i}(n, k, r, t)\right|\right\}>(1-2 \gamma) f(k / n)\left(\begin{array}{l}
n \\
k
\end{array}\right)
$$

holds for all $n>n_{3}$ and $k$ with $k / n \in I$. Finally set $n_{1}=\max \left\{n_{0}, n_{2}, n_{3}\right\}$.

Suppose that (M) fails. Then for our choice of $\varepsilon, \gamma$ and $n_{1}$, we can find some $n, k$ and $\mathscr{F} \in \mathbf{Y}^{i}(n, k, r, t)$ with $|\mathscr{F}| \geq(1-\gamma) \max \left\{\left|\mathscr{F}_{i-1}(n, k, r, t)\right|,\left|\mathscr{F}_{i}(n, k, r, t)\right|\right\}$, where $n>n_{1}$ and $\frac{k}{n} \in I$. We fix $n, k$ and $\mathscr{F}$, and let $p=\frac{k}{n}$. By (21) we have $|\mathscr{F}|>c\left(\begin{array}{l}n \\ k\end{array}\right)$, where $c=(1-$ $2 \gamma) f(p)$. Let $\mathscr{G}=\bigcup_{k \leq j \leq n}\left(\nabla_{j}(\mathscr{F})\right) \in \mathbf{X}^{i}(n, r, t)$ be the collection of all upper shadows of $\mathscr{F}$, where $\nabla_{j}(\mathscr{F})=\left\{H \in\left(\begin{array}{c}{[n]} \\ j\end{array}\right): H \supset \exists F \in \mathscr{F}\right\}$. Let $p_{1}=p+\varepsilon_{1} \in I_{0}$, and $J=n\left(p_{1} \pm \varepsilon_{1}\right)=$ $\left(k, k+2 \varepsilon_{1} n\right) \cap \mathbb{N}$.

Claim 4. $\left|\nabla_{j}(\mathscr{F})\right| \geq c\left(\begin{array}{c}n \\ j\end{array}\right)$ for $j \in J$.

Proof. Choose a real $x \leq n$ so that $c\left(\begin{array}{l}n \\ k\end{array}\right)=\left(\begin{array}{c}x \\ n-k\end{array}\right)$. Since $|\mathscr{F}|>c\left(\begin{array}{l}n \\ k\end{array}\right)=\left(\begin{array}{c}x \\ n-k\end{array}\right)$ the KruskalKatona Theorem $[21,20]$ implies that $\left|\nabla_{j}(\mathscr{F})\right| \geq\left(\begin{array}{c}x \\ n-j\end{array}\right)$. Thus it suffices to show that $\left(\begin{array}{c}x \\ n-j\end{array}\right) \geq c\left(\begin{array}{c}n \\ j\end{array}\right)$, or equivalently,

$$
\frac{\left(\begin{array}{c}
x \\
n-j
\end{array}\right)}{\left(\begin{array}{c}
x \\
n-k
\end{array}\right)} \geq \frac{c\left(\begin{array}{c}
n \\
j
\end{array}\right)}{c\left(\begin{array}{l}
n \\
k
\end{array}\right)} .
$$

Using $j \geq k$ this is equivalent to $j \cdots(k+1) \geq(x-n+j) \cdots(x-n+k+1)$, which follows from $x \leq n$.

By the claim we have

$$
w_{p_{1}}(\mathscr{G}) \geq \sum_{j \in J}\left|\nabla_{j}(\mathscr{F})\right| p_{1}^{j}\left(1-p_{1}\right)^{n-j} \geq c \sum_{j \in J}\left(\begin{array}{c}
n \\
j
\end{array}\right) p_{1}^{j}\left(1-p_{1}\right)^{n-j} .
$$

Using (20) and (19), the RHS of (22) is more than

$$
c(1-3 \gamma) /(1-2 \gamma)=(1-3 \gamma) f(p)>(1-4 \gamma) f\left(p+\varepsilon_{1}\right)=\left(1-\gamma_{0}\right) f\left(p_{1}\right) .
$$


This means $w_{p_{1}}(\mathscr{G})>\left(1-\gamma_{0}\right) \max \left\{w_{p_{1}}\left(\mathscr{G}_{i-1}(n, r, t)\right), w_{p_{1}}\left(\mathscr{G}_{i}(n, r, t)\right)\right\}$, which contradicts (W) because $p_{1} \in I_{0} \subset p_{0} \pm \varepsilon_{0}$.

Theorem 11. Let $r, t \in \mathbb{N}$ with $r \geq 4$, and let $p_{0} \in\left(0, \frac{r-3}{r-2}\right]$. Suppose that

$$
p_{0}^{t}>(t+r) p_{0}^{t+r-1}\left(1-p_{0}\right)+p_{0}^{t+r}
$$

i.e., $w_{p_{0}}\left(\mathscr{G}_{0}(n, r, t)\right)>w_{p_{0}}\left(\mathscr{G}_{1}(n, r, t)\right)$ for all $n \geq t+r$. Then (W0) implies (M1) and (W1).

(W0) There exist positive constants $\gamma_{0}, \varepsilon_{0}, n_{0}$ such that $w^{0}(n, p, r, t)<\left(1-\gamma_{0}\right) p^{t}$ holds for all $p$ with $\left|p-p_{0}\right|<\varepsilon_{0}$ and all $n$ with $n \geq n_{0}$.

(M1) There exist positive constants $\gamma_{1}, \varepsilon_{1}, n_{1}$ such that

$$
m^{0}(n, k, r, t)<\left(1-\gamma_{1}\right)\left(\begin{array}{c}
n-t \\
k-t
\end{array}\right)
$$

holds for all $n>n_{1}$ and $k$ with $\frac{k}{n}<p_{0}+\varepsilon_{1}$.

(W1) There exist positive constants $\gamma_{2}, \varepsilon_{2}$ such that $w^{0}(n, p, r, t)<\left(1-\gamma_{2}\right) p^{t}$ holds for all $p$ with $p<p_{0}+\varepsilon_{2}$ and all $n$ with $n \geq t$.

Proof. For simplicity, we write $\mathscr{G}_{j}$ for $\mathscr{G}_{j}(n, r, t)$ and $\mathscr{F}_{j}$ for $\mathscr{F}_{j}(n, k, r, t)$.

Assume (W0). First we show (M1). Choose $\varepsilon_{0}$ from (W0). Since $w_{p_{0}}\left(\mathscr{G}_{0}\right)>w_{p_{0}}\left(\mathscr{G}_{1}\right)$ we may assume that $w_{p}\left(\mathscr{G}_{0}\right)>w_{p}\left(\mathscr{G}_{1}\right)$ for all $p$ with $\left|p-p_{0}\right|<\varepsilon_{0}$ (if necessary we replace $\varepsilon_{0}$ so that this property holds). We can choose $n_{1}$ so that $\left|\mathscr{F}_{0}\right|>\left|\mathscr{F}_{1}\right|$ holds for all $n>n_{1}$ and $k$ with $\left|\frac{k}{n}-p_{0}\right|<\varepsilon_{0}$. Then for the parameters chosen as above, we have $w^{0}(n, p, r, t)=$ $w^{1}(n, p, r, t)$ and $m^{0}(n, k, r, t)=m^{1}(n, k, r, t)$. Thus (23) for the case $\left|\frac{k}{n}-p_{0}\right|<\varepsilon_{1}:=\frac{\varepsilon_{0}}{2}$ follows from Theorem 10 by setting $i=1$. We will show (23) for $\frac{k}{n} \leq p_{0}-\varepsilon_{1}$. Let $p=p_{0}-\frac{\varepsilon_{1}}{2}$. Since $p<p_{0}$ and $w_{p}\left(\mathscr{G}_{0}\right)=p^{t}>w_{p}\left(\mathscr{G}_{1}\right)$ we can choose $\gamma_{1}>0$ so that

$$
\left(1-2 \gamma_{1}\right) p^{t}>w_{p}\left(\mathscr{G}_{1}(n, r, t)\right) \text {. }
$$

Then choose $n_{0}$ so that

$$
\sum_{i \in J}\left(\begin{array}{c}
n-t \\
i-t
\end{array}\right) p^{i}(1-p)^{n-i}>p^{t}\left(1-2 \gamma_{1}\right) /\left(1-\gamma_{1}\right)
$$

holds for all $n>n_{0}$, where $J=n\left(p \pm \frac{\varepsilon_{1}}{2}\right)=\left(\left(p_{0}-\varepsilon_{1}\right) n, p_{0} n\right) \cap \mathbb{N}$.

To show (23), suppose, on the contrary, that we can find some $n, k$ and $\mathscr{F} \in \mathbf{Y}^{0}(n, k, r, t)$ with $|\mathscr{F}| \geq\left(1-\gamma_{1}\right)\left(\begin{array}{c}n-t \\ k-t\end{array}\right)$, where $n>n_{1}$ and $\frac{k}{n} \leq p_{0}-\varepsilon_{1}$. We fix $n, k$ and $\mathscr{F}$. Let $\mathscr{G}=$ $\bigcup_{k \leq i \leq n}\left(\nabla_{i}(\mathscr{F})\right) \in \mathbf{X}^{0}(n, r, t)$ be the collection of all upper shadows of $\mathscr{F}$.

Claim 5. $\left|\nabla_{i}(\mathscr{F})\right| \geq\left(1-\gamma_{1}\right)\left(\begin{array}{c}n-t \\ i-t\end{array}\right)$ for $i \in J$.

Proof. Choose a real $x \leq n-t$ so that $\left(1-\gamma_{1}\right)\left(\begin{array}{c}n-t \\ k-t\end{array}\right)=\left(\begin{array}{c}x \\ n-k\end{array}\right)$. Since $|\mathscr{F}| \geq\left(\begin{array}{c}x \\ n-k\end{array}\right)$ the Kruskal-Katona Theorem implies that $\left|\nabla_{i}(\mathscr{F})\right| \geq\left(\begin{array}{c}x \\ n-i\end{array}\right)$. Thus it suffices to show that $\left(\begin{array}{c}x \\ n-i\end{array}\right) \geq\left(1-\gamma_{1}\right)\left(\begin{array}{c}n-t \\ i-t\end{array}\right)$, or equivalently,

$$
\frac{\left(\begin{array}{c}
x \\
n-i
\end{array}\right)}{\left(\begin{array}{c}
x \\
n-k
\end{array}\right)} \geq \frac{\left(1-\gamma_{1}\right)\left(\begin{array}{c}
n-t \\
i-t
\end{array}\right)}{\left(1-\gamma_{1}\right)\left(\begin{array}{l}
n-t \\
k-t
\end{array}\right)}
$$

Using $i>\left(p_{0}-\varepsilon_{1}\right) n \geq k$ this is equivalent to $(i-t) \cdots(k-t+1) \geq(x-n+i) \cdots(x-n+$ $k+1$ ), which follows from $x \leq n-t$. 
By the claim we have

$$
w_{p}(\mathscr{G}) \geq \sum_{i \in J}\left|\nabla_{i}(\mathscr{F})\right| p^{i}(1-p)^{n-i} \geq\left(1-\gamma_{1}\right) \sum_{i \in J}\left(\begin{array}{c}
n-t \\
i-t
\end{array}\right) p^{i}(1-p)^{n-i} .
$$

By (25) and (24), the RHS of (26) is more than $\left(1-\gamma_{1}\right) \cdot p^{t}\left(1-2 \gamma_{1}\right) /\left(1-\gamma_{1}\right)=p^{t}(1-$ $\left.2 \gamma_{1}\right)>w_{p}\left(\mathscr{G}_{1}(n, r, t)\right)$, which contradicts (W0). This completes the proof of (M1).

Next we show (W1). Let $\varepsilon_{1}=\frac{\varepsilon_{0}}{2}$ and let $p \leq p_{0}-\varepsilon_{1}$ be given. By (M1) we can find $\gamma_{1}>0$ and $n_{1}$ such that $m^{0}(n, k, r, t)<\left(1-\gamma_{1}\right)\left(\begin{array}{l}n-t \\ k-t\end{array}\right)$ holds for all $n>n_{1}$ and $k$ with $\frac{k}{n}<p_{0}$. Choose $0<\delta \ll \varepsilon_{1}$ so that $p \pm \delta \subset\left(0, p_{0}\right)$. Choose $n_{2}$ so that

$$
\left(1-\gamma_{1}\right) \sum_{k \in J}\left(\begin{array}{l}
n-t \\
k-t
\end{array}\right) p^{k} q^{n-k}+\sum_{k \notin J}\left(\begin{array}{l}
n \\
k
\end{array}\right) p^{k} q^{n-k}<\left(1-\frac{\gamma_{1}}{2}\right) p^{t}
$$

holds for all $n>n_{2}$, where $J=n(p \pm \delta)$. Let $n>\max \left\{n_{1}, n_{2}\right\}$ and choose $\mathscr{G} \in \mathbf{X}^{0}(n, r, t)$ with $w_{p}(\mathscr{G})=w^{0}(n, p, r, t)$. Let $\mathscr{G}^{(k)}=\mathscr{G} \cap\left(\begin{array}{c}{[n]} \\ k\end{array}\right)$ for $k \in J$.

If $\mathscr{G}^{(k)} \in \mathbf{Y}^{0}(n, k, r, t)$ then we have $\left|\mathscr{G}^{(k)}\right| \leq m^{0}(n, k, r, t)<\left(1-\gamma_{1}\right)\left(\begin{array}{l}n-t \\ k-t\end{array}\right)$. If $\mathscr{G}^{(k)}$ fixes $t$ vertices, say $[t]$, then $\tilde{\mathscr{G}}^{(k)}:=\left\{G-[t]: G \in \mathscr{G}^{(k)}\right\}$ is $(r-1)$-wise 1-intersecting. (Otherwise $\mathscr{G}$ fixes $[t]$.) Thus we have $\left|\mathscr{G}^{(k)}\right|=\left|\tilde{\mathscr{G}}^{(k)}\right| \leq\left(\begin{array}{c}n-t-1 \\ k-t-1\end{array}\right)=\frac{k-t}{n-t}\left(\begin{array}{c}n-t \\ k-t\end{array}\right)<p_{0}\left(\begin{array}{c}n-t \\ k-t\end{array}\right)$ by (2). Consequently, in both cases, we have

$$
\left|\mathscr{G}^{(k)}\right|<\left(1-\gamma_{1}\right)\left(\begin{array}{c}
n-t \\
k-t
\end{array}\right) .
$$

Using (28) and (27), we have

$$
w_{p}(\mathscr{G}) \leq \sum_{k \in J}\left|\mathscr{G}^{(k)}\right| p^{k} q^{n-k}+\sum_{k \notin J}\left(\begin{array}{l}
n \\
k
\end{array}\right) p^{k} q^{n-k}<\left(1-\frac{\gamma_{1}}{2}\right) p^{t},
$$

and this is true for all $n \geq t$ by Lemma 6 . This completes the proof of (W1).

By Theorems 8, 10 and 11, we have the following.

Theorem 12. Let $r \geq 4$. There exists $n_{1}$ such that

$$
m(n, k, r, t)=\max \left\{\left|\mathscr{F}_{0}(n, k, r, t)\right|,\left|\mathscr{F}_{1}(n, k, r, t)\right|\right\}
$$

holds for all $t$ with $1 \leq t \leq\left(3^{r}-2 r-1\right) / 2$, and for all $n>n_{1}$ and $k$ with $\frac{k}{n}<\frac{1}{3}+\varepsilon$. Moreover $\mathscr{F}_{0}(n, k, r, t)$ and $\mathscr{F}_{1}(n, k, r, t)$ are the only possible optimal configurations (up to isomorphism).

\section{REFERENCES}

[1] R. Ahlswede, L.H. Khachatrian. The complete nontrivial-intersection theorem for systems of finite sets. J. Combin. Theory (A), 76:121-138, 1996.

[2] R. Ahlswede, L.H. Khachatrian. The complete intersection theorem for systems of finite sets. European J. Combin., 18:125-136, 1997.

[3] R. Ahlswede, L.H. Khachatrian. The diametric theorem in Hamming spaces - Optimal anticodes. Adv. in Appl. Math., 20:429-449, 1998.

[4] A. Brace, D. E. Daykin. A finite set covering theorem. Bull. Austral. Math. Soc., 5:197-202, 1971.

[5] C. Bey, K. Engel. Old and new results for the weighted $t$-intersection problem via AK-methods. Numbers, Information and Complexity, Althofer, Ingo, Eds. et al., Dordrecht, Kluwer Academic Publishers, 45-74, 2000.

[6] P. Erdős, C. Ko, R. Rado. Intersection theorems for systems of finite sets. Quart. J. Math. Oxford (2), 12:313-320, 1961.

[7] I. Dinur, S. Safra. On the Hardness of Approximating Minimum Vertex-Cover. Annals of Mathematics, 162:439-485, 2005. 
[8] P. Frankl. On Sperner families satisfying an additional condition. J. Combin. Theory (A), 20:1-11, 1976.

[9] P. Frankl. Families of finite sets satisfying an intersection condition. Bull. Austral. Math. Soc., 15:7379, 1976.

[10] P. Frankl. The Erdős-Ko-Rado theorem is true for $n=c k t$. Combinatorics (Proc. Fifth Hungarian Colloq., Keszthey, 1976), Vol. I, 365-375, Colloq. math. Soc. János Bolyai, 18, North-Holland, 1978.

[11] P. Frankl. On intersecting families of finite sets. J. Combin. Theory (A), 24:141-161, 1978.

[12] P. Frankl. The shifting technique in extremal set theory. "Surveys in Combinatorics 1987" (C. Whitehead, Ed. LMS Lecture Note Series 123), 81-110, Cambridge Univ. Press, 1987.

[13] P. Frankl. Multiply-intersecting families. J. Combin. Theory (B), 53:195-234, 1991.

[14] P. Frankl, N. Tokushige. Weighted 3-wise 2-intersecting families. J. Combin. Theory (A) 100:94-115, 2002.

[15] P. Frankl, N. Tokushige. Weighted multiply intersecting families. Studia Sci. Math. Hungarica 40:287291, 2003.

[16] P. Frankl, N. Tokushige. Random walks and multiply intersecting families. J. Combin. Theory (A), 109:121-134, 2005.

[17] P. Frankl, N. Tokushige. Weighted non-trivial multiply intersecting families. Combinatorica, 26 37-46, 2006.

[18] A. J. W. Hilton, E. C. Milner. Some intersection theorems for systems of finite sets. Quart. J. Math. Oxford, 18:369-384, 1967.

[19] G.O.H. Katona. Intersection theorems for systems of finite sets. Acta Math. Acad. Sci. Hung., 15:329337, 1964.

[20] G.O.H. Katona. A theorem of finite sets, in: Theory of Graphs, Proc. Colloq. Tihany, 1966 (Akademiai Kiadó, 1968) 187-207.

[21] J.B. Kruskal. The number of simplices in a complex, in: Math. Opt. Techniques (Univ. of Calif. Press, 1963) 251-278.

[22] N. Tokushige. A frog's random jump and the Pólya identity. Ryukyu Math. Journal, 17:89-103, 2004.

[23] N. Tokushige. Intersecting families — uniform versus weighted. Ryukyu Math. J., 18:89-103, 2005.

[24] N. Tokushige. Extending the Erdős-Ko-Rado theorem. J. Combin. Designs, 14:52-55, 2006.

[25] N. Tokushige. The maximum size of 4-wise 2-intersecting and 4-wise 2-union families. European J. of Comb., 27:814-825, 2006.

[26] N. Tokushige. The maximum size of 3-wise $t$-intersecting families. European J. Combin, 28:152-166, 2007.

[27] N. Tokushige. EKR type inequalities for 4-wise intersecting families. J. Combin. Theory (A), 114:575596, 2007.

[28] N. Tokushige. Brace-Daykin type inequalities for intersecting families. European J. Combin, 29:273285, 2008.

[29] N. Tokushige. Multiply-intersecting families revisited. J. Combin. Theory (B), 97:929-948, 2007.

[30] R.M. Wilson. The exact bound in the Erdős-Ko-Rado theorem. Combinatorica, 4:247-257, 1984.

College of Education, Ryukyu University, Nishihara, OKINAWA, 903-0213 JAPAN

E-mail address: hide@edu.u-ryukyu.ac.jp 